OPEN ACCESS

Citation: Monica Dati (2021) 150 ore e sperimentazione didattica: il caso del teatro operaio a Terni. Rivista di Storia dell'Educazione 8(1): 49-59. doi: $10.36253 /$ rse- 10014

Received: November 13, 2020

Accepted: April 16, 2021

Published: July 5, 2021

Copyright: @ 2021 Monica Dati. This is an open access, peer-reviewed article published by Firenze University Press (http://www.fupress.com/rse) and distributed under the terms of the Creative Commons Attribution License, which permits unrestricted use, distribution, and reproduction in any medium, provided the original author and source are credited.

Data Availability Statement: All relevant data are within the paper and its Supporting Information files.

Competing Interests: The Author(s) declare(s) no conflict of interest.

Editor: Pietro Causarano, Università di Firenze.

\section{0 ore e sperimentazione didattica: il caso del teatro operaio a Terni}

\author{
150 hours and teaching experimentation: the case of Terni \\ workers' theater
}

\author{
Monica Dati \\ Università di Firenze \\ E-mail: monica.dati@unifi.it
}

\begin{abstract}
The paper has the porpose to explain how the contractual statute of the150hours promoted an interesting reinterpretation of culture and schooling and how the practical activities of the courses were based on the experience of workers. An overview to contextualize the Terni workers' case who decided to use the paid hours to explore the importance of the theater as an educational and communication tool to transform the social reality. A report of a unique experience made possible by the support of LiberEtà Cgil which has provided the contact of the coordinator of the theater workshop, Gian Filippo Della Croce (Fiom).
\end{abstract}

Keywords: 150 hours, theater, adult education.
Riassunto. Larticolo prende in considerazione come l'istituto contrattuale delle 150 ore promosse una stimolante rilettura della cultura e della scolarizzazione e come le attività concrete dei corsi si proponessero di facilitare l'incontro tra l'esperienza dei lavoratori e il sapere di cui la scuola, sia pure di tipo "nuovo", si faceva garante. Una panoramica utile a contestualizzare quanto riportato nell'approfondimento dedicato ai lavoratori delle acciaierie di Terni che decisero di utilizzare il monte ore retribuito per impadronirsi del teatro come mezzo didattico e di comunicazione per partecipare al processo di trasformazione della realtà sociale. Vicenda unica e carica di significati alla cui restituzione ha dato un fondamentale contributo LiberEtà Cgil che ha fornito il contatto di uno dei protagonisti, Gian Filippo Della Croce (Fiom), coordinatore del seminario, di cui si riportano alcuni stralci di intervista.

Parole chiave: 150 ore, teatro, educazione degli adulti.
Ma che ve ne fate delle 150 ore? Imparerete a suonare il clavicembalo? Se sarà necessario perché no ${ }^{1}$

\footnotetext{
${ }^{1}$ Episodio "mitizzato" della trattativa del 1973. Walter Mandelli allora presidente di Federmeccanica, di fronte alla richiesta di utilizzo delle 150 ore in senso ampio e svincolato da aspetti professio-
} 


\section{ORE ED ISTANZE DI RINNOVAMENTO TRA CULTURA, FABBRICA E SCUOLA}

Gli anni Settanta rappresentarono per l'Italia un decennio di sperimentazione straordinaria sul piano culturale e sociale, la scuola stessa si andava trasformando e si avviavano programmi per la diffusione capillare della cultura. Uno strumento rivoluzionario in questo senso fu l'introduzione delle 150 ore di studio per i lavoratori: nel 1973, con l'art. 28 del Ccnl metalmeccanici venne infatti riconosciuto ai dipendenti il diritto a permessi retribuiti per frequentare, presso istituti pubblici o legalmente riconosciuti, corsi di studio al fine di migliorare la propria cultura.

La conquista del monte ore retribuito (utilizzato per ottenere il diploma di terza media, frequentare seminari o insegnamenti monografici nella scuola secondaria superiore e all'Università) con la sua carica di sperimentazione e approfondimento del rapporto studio-lavoro si inseriva in un contesto enormemente dinamico e complesso. La storia d'Italia tra la fine degli anni ' 60 e l'inizio degli anni '70 fu caratterizzata, come noto, da una lunga serie di scontri che videro in prima linea studenti ed operai battersi contro molteplici contraddizioni economiche, politiche e sociali. L'Autunno caldo del ' 69 , preceduto ma non separato dalle contestazioni studentesche del ' 68 , si inquadrò in un clima infuocato dagli squilibri dello sviluppo e della modernizzazione che aprirono nuovi scenari, suscitando aspettative crescenti che rapidamente si valsero in delusione e frustrazione. Al centro la scuola e la fabbrica. Modifiche dei ritmi produttivi divenuti insostenibili, riduzione dell'orario di lavoro, superamento della divisione «voluta dal padrone tra lavoratori intellettuali e lavoratori manuali» (Gurrieri 1975 , 11) miglioramento delle condizioni di salute e sicurezza nelle fabbriche erano le richieste che avanzavano gli operai. Lavoratori spesso dequalificati, non di mestiere, ma soprattutto esclusi dai percorsi scolastici. Una questione che il dibattito studentesco aveva saputo mettere ben in evidenza: il conflitto attorno alle risorse culturali e all'accesso ad esse. Nelle piazze e nelle scuole si contestava l'autoritarismo delle strutture accademiche e la riduzione nozionistica della cultura ma anche l'incoerenza del modello economico capitalistico rispetto al pieno dispiegamento delle opportunità e potenzialità individuali e il perdurare di una scuola classista che presiedeva alla selezione e alla mobilità sociali, attraverso l'istruzione e poi la condizione professionale. Del res-

nali, pose provocatoriamente questa domanda ai sindacati. Il clavicembalo si radicò poi nell'immaginario collettivo grazie alla copertina del numero speciale e congiunto delle riviste "Fabbrica e Stato" e "Inchiesta" del 1973, intitolato "Suonata per i padroni". to la riforma della scuola media del '62 era già stata, in un certo senso, l'emblema di una scolarizzazione di massa che era tale più in entrata che in uscita come denunciato da Barbagli e Dei e dal libro manifesto "Lettera ad una professoressa" (Santamaita 2010). Una questione che dal punto di vista dei lavoratori si sarebbe tradotta nella denuncia dell'incapacità del sistema scuola-lavoro di rispondere ai mutati bisogni sociali, sul piano individuale e collettivo; dal punto di vista sindacale nel porre il nesso tra diritto allo studio, qualità effettiva sul lavoro e governo della mobilità professionale in azienda. La divisione in fabbrica nasceva a scuola e si trattava di riconoscere a tutti i lavoratori non solo il diritto di accedervi e fruirne ma anche di contribuire a trasformarla, gestirla, rifondarla (Lettieri 1970).

Le 150 ore rappresentarono pertanto qualcosa di innovativo e culturalmente dirompente. La conquista del monte ore retribuito riuscì a creare dei veri e propri spazi di tempo libero dentro il rigido orario di lavoro realizzando richieste già avvertite per tutti gli anni ‘60 dal movimento dei lavoratori studenti, costretti a giocare le loro chances di elevazione culturale e professionale sacrificando la sfera della vita privata (Foa 1969). Il nuovo istituto realizzò un cambiamento enorme anche rispetto ad una tradizione di intervento, sia pubblico che privato, di origine prevalentemente assistenziale se non filantropica e sempre concepita come compensativa rispetto al deficit di formazione dei gruppi sociali subalterni: «eravamo certi che l'educazione degli adulti non fosse per nulla un aspetto del filantropismo della borghesia laica o religiosa ma il risultato delle pressioni e delle lotte della classe operaia per affermare, anche nel campo culturale la propria autonomia» (De Sanctis 1978, 335).

Di fatto si trattò di un vero e proprio laboratorio collettivo di sperimentazione per le innovazioni didattiche e organizzative nell'educazione degli adulti, alternativo alla scuola del mattino fatta di voti, insegnanti autoritari, compiti a casa, dai contenuti astratti e lontani dall'esperienza quotidiana: un'ulteriore scommessa da parte del movimento operaio e sindacale riguardo alla democratizzazione di una scuola ancora selettiva ed elitaria, che traeva vigore dall'influenza del movimento studentesco, dall'esperienza di Don Milani a Barbiana, come da altri fermenti innovatori che si erano delineati ad inizio degli anni '60. In un contesto di assenza di un vero e qualificato luogo istituzionale per la formazione degli adulti, di un necessario rinnovamento e di una democratizzazione della scuola, le 150 ore si imposero come un'esperienza ben distinta e innovativa rispetto alla tradizione della vecchia educazione popolare, per molti aspetti intrisa di paternalismo e assistenzialismo. 
Metodologie didattiche nuove che aprivano orizzonti e percorsi inediti, superando l'atteggiamento passivo nei confronti dello studio di cui è emblematico esempio l'esperienza del teatro operaio a Terni: i lavoratori delle acciaierie portarono in scena nel 1975 uno dei drammi didattici di Bertolt Brecht, "L'eccezione e la regola", scritta dal drammaturgo tedesco tra il 1929 e il 1930 con il preciso scopo di attivare la partecipazione degli operai attraverso il teatro.

In quegli anni c'era in giro un risveglio culturale innovativo, propositivo, anche rivoluzionario. Tale condizione appariva soprattutto nel teatro dove si moltiplicavano le formazioni teatrali di base, le compagnie cooperative, dove si riscopriva la funzione innovativa o rivoluzionaria di un mezzo che per troppo tempo era stato proprietà della borghesia. Far entrare il teatro in fabbrica significava dare ai lavoratori e alla fabbrica un nuovo protagonismo sociale ${ }^{2}$.

Come ricordano le parole di Gian Filippo Della Croce, coordinatore del seminario, gli anni Settanta rappresentarono un momento di sperimentazione di nuovi modi di produrre e organizzare la cultura, al di fuori dei parametri delle istituzioni. In campo teatrale l'attenzione si spostò ai "margini" della scena ufficiale, nei luoghi dove le contraddizioni sociali e i dissensi erano più acuti (Bernardi 2014). Il teatro doveva farsi per le strade, fra la gente, proponendosi come mezzo di agitazione e impegno sociale negando l'arte come fatto professionistico e

\footnotetext{
${ }^{2}$ Dall'intervista a Gian Filippo Della Croce (sindacalista FIOM CGIL), nato a Terni nel 1942 e coordinatore del seminario 150 ore alle acciaierie nel 1975. L'intervista è stata realizzata a distanza il 30 settembre del 2020 e si inserisce all'interno di un più ampio progetto di ricostruzione dellesperienza delle 150 ore attraverso testimonianze autobiografiche di prossima pubblicazione. A fornire i contatti dell'intervistato è stata LiberEtà Cgil nell'ambito del premio "Guido Rossa" al quale Della Croce ha partecipato con l'opera "Gli operai cantano". Di seguito si riporta la scaletta di intervista: In quale contesto storico-sociale è entrato in contatto con le 150 ore? Utilizzare le 150 ore per il teatro acquisiva significati particolari? Come fu accolta l'iniziativa del seminario su Brecht da azienda, consiglio di fabbrica ed operai? Dove e come si svolse il seminario? Ha aneddoti particolari da raccontare? Attraverso questa esperienza ci fu una presa di coscienza da parte degli operai (come negli intenti dei drammi didattici di Brecht)? Da questa vicenda cosa appresero? Gli operai che parteciparono avevano tutti conseguito la terza media? Che titoli di studi avevano? Che mansioni svolgevano? Ci furono critiche legate al fatto di spendere le 150 ore per il teatro e non per un utilizzo più classico dell'istituto come il conseguimento del diploma? Come valuta globalmente la vicenda? "Leccezione e la regola", testo didattico, può svolgere ancora oggi la funzione per la quale Brecht lo scrisse? Potrebbe essere replicabile anche oggi? Leducazione degli adulti oggi sembra acquisire una connotazione marcatamente funzionalistica asservita alle necessità del lavoro, alla luce della sua esperienza legata al teatro operaio potrebbe essere utile introdurre percorsi di studio umanistici all' interno delle aziende? Perchè? Che opinione ha delle 150 ore? Come valuta questa esperienza? Quali sono secondo il suo giudizio gli elementi che portarono alla loro fine? Ha senso, ancora oggi, parlare e discutere di 150 ore nei contesti di fabbrica?
}

dando la parola a soggetti normalmente non rappresentati per allargare il diritto all'espressione e alla creatività. Richieste di rinnovamento che prendevano ispirazione anche dal Teatro dell'Oppresso di Augusto Boal che, seguace delle teorie di Brecht, intravedeva nella rappresentazione scenica un mezzo di presa di coscienza e trasformazione della realtà. Queste premesse furono alla base di molteplici esperienze che avvicinarono il teatro alla vita degli individui e delle comunità: nelle scuole grazie agli influssi del Movimento di Cooperazione Educativa, nelle carceri (Taormina, Valenti, 2013) e negli ospedali psichiatrici ${ }^{3}$ ma anche nelle fabbriche.

L'esperienza vissuta dagli operai di Terni esprimeva in modo significativo queste istanze e sottolineava con forza la volontà di proporre una sorta di controcultura operaia antagonista a quella dell'impresa e alla "scienza dei padroni". Usare le 150 ore per il teatro rappresentava inoltre il superamento di una visione aziendalistica del diritto alla studio, lo scopo principale dell'art. 28 infatti non era tanto il miglioramento delle capacità produttive del lavoratore bensì il loro arricchimento culturale come ebbe modo di evidenziare Lucio Pagnoncelli:

Si affermano nuovi valori e una nuova concezione circa il rapporto stesso cultura-lavoro e circa la socialità di questo rapporto. Se infatti l'azienda si impegna a retribuire il tempo di lavoro utilizzato dal lavoratore per svolgere attività di studio che ha scelto liberamente e se lo Stato, attraverso il sistema pubblico di istruzione, si impegna a finanziare corsi che i lavoratori avranno stabilito di seguire, si viene implicitamente a riconoscere per un verso la rilevanza in termini di positive ripercussioni sul lavoro, della crescita culturale dei lavoratori, qualunque essa sia; per l'altro la socialità del diritto allo studio, sia nei termini passivi di garanzia sociale per l'accesso a tale diritto, sia nei termini attivi del valore di arricchimento collettivo determinato dalla crescita culturale. Non sono questioni da poco se teniamo presente la forte arretratezza del dibattito sul rapporto studio-lavoro nel nostro paese e i termini angusti e spesso meccanicistici in cui si muove; né d'altra parte va sottovalutata l'importanza di queste indicazioni ai fini di una più complessiva ristrutturazione del sistema educativo nella direzione indicata dalle proposte di educazione permanente e ricorrente (Pagnoncelli 1977, 24-25).

Il significato di questa inedita battaglia sul piano politico ed educativo non si fermava alla sola necessità di aggiornamento e formazione culturale degli operai ma risiedeva anche nella volontà di trasformazione in chiave

\footnotetext{
${ }^{3}$ Sugli ospedali psichiatrici si ricorda il laboratorio teatrale allestito da Giuliano Scabia con i ricoverati di Trieste nel 1972 che portò alla costruzione di un cavallo di cartapesta fatto sfilare per le strade della città e poi in tanti altri luoghi (Cuppone 2018).
} 
partecipativa ed emancipatoria della realtà di fabbrica e della classe operaia. Un' occasione per riflettere sul ruolo delle cosiddette Humanities nei contesti lavorativi (Cornacchia 2018) e per sottolineare come i processi di apprendimento in età adulta non debbano essere esclusivamente asserviti a logiche funzionaliste e al «riciclaggio tecnologico-professionale dei lavoratori» (Santamauro 1975). La dimensione collettiva delle 150 ore è infatti sfiorita nelle attese individuali, spesso frustate e non facilmente negoziabili sui luoghi di lavoro, sempre più focalizzate su bisogni strumentali e di aggiornamento tecnico:

Certo l'idea di una formazione indipendente dagli scopi immediatamente professionali, germe di elaborazione culturale della persona e della classe, l'idea insomma che il tempo di studio possa avere valore sociale e persino essere materia di contrattazione anche per suonare il clavicembalo ha ormai perso senso. Il discorso educativo odierno è tutto volto allo scopo di accrescere le proprie possibilità sul mercato del lavoro, il che non significa affatto che le persone non si appassionino più al clavicembalo: semplicemente a nessuno viene in mente di difendere il significato politico e sociale di una simile scelta. Si tratta di un hobby, di tempo libero reso fruttuoso, di un affare del tutto privato e quindi socialmente e politicamente irrilevante (Causarano, Zazzara 2015, 10).

\section{ORE: UN LABORATORIO DI SPERIMENTAZIONE PER L'EDUCAZIONE DEGLI ADULTI}

Componente significativa del modello 150 ore era la richiesta di un proprio impianto culturale in contrapposizione con la scuola tradizionale, che doveva esserne a sua volta influenzata ed ispirata. Una volta scartata la soluzione della scuola popolare, diveniva fondamentale l'elaborazione di contenuti propri, diversi dai programmi del mattino o del corso di addestramento e aggiornamento aziendale. I corsi, di scuola media in primis, richiesero pertanto ad insegnanti, lavoratori, quadri sindacali, un vasto impegno di individuazione ed elaborazione di nuovi contenuti e metodi didattici. La necessità di tener conto dell'esperienza sindacale e politica dei lavoratori, di collegarla e confrontarla con la cultura in un rapporto di apprendimento motivante e in linea con le esigenze di un discente adulto, comportava una revisione dei modelli culturali vigenti nella scuola media, una modifica del ruolo dell'insegnante, l'abbandono di alcuni tradizionali strumenti didattici: ciò che mancava palesemente era un sistema qualificato di educazione degli adulti. La legge Gonnella e i corsi Cracis rispecchiavano una ripetizione ancora più degradata della scuola del mattino e non un vero e qualificato luogo istituzion- ale per la formazione di base degli adulti: era evidente che le 150 ore non potevano rappresentare per i lavoratori un ritorno nella stessa scuola con voti, insegnanti autoritari, compiti a casa, dai contenuti astratti e lontani dalla loro esperienza quotidiana.

I tempi stavano cambiando: l'educazione permanente si stava diffondendo e si affermavano «teorie ed esperienze di educazione degli adulti volte a valorizzare l'educabilità di ognuno, ad incoraggiare la partecipazione, ad accreditare il diritto e la possibilità di ogni uomo di crescere culturalmente, di arricchire e potenziare la sua umanità in ogni momento della sua esistenza e di produrre cultura» (Targhetta 2015, 31). La molteplicità di attori sociali e movimenti che scosse la società italiana tra gli anni Sessanta e Settanta impose il tema dell'istruzione al centro di un ampio progetto di rinnovamento politico e sociale. Il movimento a favore della scuola popolare, le riviste ideologicamente orientate (come L'erba voglio) e molti altri soggetti contribuirono a gettare un tassello nel rinnovamento e democratizzazione della scuola. Nel caso delle 150 ore era inoltre necessario rispondere ad una richiesta culturale nuova, collegata agli specifici obiettivi delle lotte operaie, ai problemi dell'organizzazione del lavoro, alla linea egualitaria portata avanti dalla Flm (Federazione lavoratori metalmeccanici) tra il '72 e il '73. "I lavoratori sono entrati nella scuola per cambiarla", veniva ribadito in tanti documenti sindacali e si trattava di tradurre questo obiettivo, questa volontà di rinnovamento, nell' effettiva elaborazione, di nuovi contenuti, nella costruzione di piani di studio, in concrete scelte metodologiche e didattiche. Innanzitutto si trattava di valorizzare l'esperienza di lavoro.

Nelle indicazioni sui programmi di molte federazioni (Bologna, Reggio Emilia, Varese, Genova) l'esperienza era intesa soprattutto come esperienza lavorativa: in questo senso si impose decisamente la centralità della fabbrica. Il programma di Bologna per esempio poneva come "punto di riferimento costante», «l'organizzazione del lavoro in tutti i suoi risvolti (storici, sociali, economici, tecnico- scientifici)» e «le singole discipline intervengono come supporto alla conoscenza concreta di questo filone fondamentale» (Rossetti Pepe 1975, 90). Per quanto riguarda la proposta di Milano, l'esperienza si riferiva più largamente al vissuto dei lavoratori, alla storia personale, e includeva esperienze come la scuola, l'emigrazione, il trasferimento dalla campagna alla città ecc. In tutti i casi il passaggio dal particolare della esperienza individuale alla comprensione della dimensione generale dei problemi avveniva attraverso la ricerca e lo studio della storia: "Nella scuola tradizionale la storia viene intesa come una sfilza di date, nomi e cifre: per noi la storia è l'occasione per sapere non le gesta dei 
poeti, santi ed eroi ma per sapere invece le oscura gesta di milioni di lavoratori che sono i soli che attraverso la lotta di classe hanno modificato la società e quindi dato un'impronta a tutta la storia» ${ }^{4}$.

Anche all'interno dei corsi 150 ore del 1974 svolti alla Bovisa nell'ambito della sperimentazione didattica tentata su un ampio ventaglio di aziende presenti sul territorio milanese si sollecitava l'interesse dei corsisti a partire dalla loro concreta esperienza di vita. Così, ad esempio, per la matematica, si iniziava l'insegnamento dalla capacità di comprendere a fondo il sistema salariale e di remunerazione, a cominciare dalla busta paga e dalla sua struttura. Vittorio Foa, nei corsi organizzati dalla Flm per il recupero della licenza media, si impegnò direttamente tenendo lezioni sulla lettura del cedolino poi pubblicate nel testo "La struttura del Salario" (1976). Per la storia e la geografia si puntava all'esperienza traumatica dell'emigrazione o sui ricordi del fascismo mentre non erano affatto rari i casi in cui nell'ambito dell'insegnamento dell'italiano si prevedesse una vera e propria fase di alfabetizzazione primaria e funzionale. Del resto i dati ufficiali del censimento del 1971 denunciavano un tasso di analfabetismo tra la popolazione italiana pari al $32,4 \%$ e la sola industrializzata Lombardia contava un milione e ottocentomila adulti analfabeti o privi di titolo di studio. Sembrava pertanto politicamente doveroso, così come urgente, pensare ad una mobilitazione democratica anche per tutti gli adulti e lavoratori senza alfabeto, più esclusi ed emarginati anche per il solo fatto di essere incapaci di leggere volantini e manifesti utilizzati per il reperimento dei corsi 150 ore. Nelle lezioni per lavoratori spesso veniva fatto uso del quotidiano per la facilità che presentava la sua lettura, offrendo al lavoratore l'opportunità di maturare capacità critica per smontare i meccanismi persuasivi della lingua attraverso l'esame di titoli, impaginazione, fotografie, testi di cronaca.

L'introduzione del giornale nella scuola non è più un fatto scandaloso; anni di lotte studentesche hanno reso plausibile questa possibilità. Resta comunque nelle autorità scolastiche una certa resistenza per tale innovazione: il giornale è considerato estraneo ai programmi, il giornale insegna un cattivo italiano (che non è quello delle belle lettere), il giornale introduce la politica e peggio, i partiti nella scuola; ma queste obiezioni possono essere controbattute, sono i programmi ministeriali a essere estranei alla vita reale, è la lingua scolastica ad essere altra cosa dalla lingua quotidiana, e ben venga la conoscenza della politica, dei partiti, della storia d'oggi. Leggere il giornale significa aprire la scuola al mondo e ai suoi problemi (Cavazzoni 1976, 14).

\footnotetext{
4 "A scuola con le 150 ore", Flm Lambrate, Fondo Flm, b.13873 fasc. 2
}

Altri cambiamenti dovevano riguardare per l'appunto il metodo e gli strumenti didattici. Le indicazioni sindacali proseguirono sul rifiuto del libro optando per la preparazione di materiali specifici (quotidiani, proiezioni video) e la costituzione di biblioteche di corso, oltre a fornire ai corsisti manuali ciclostilati legati agli argomenti di studio, da adoperare individualmente. Per quanto concerne il metodo l'attenzione fu posta sulla discussione, sul lavoro di gruppo, la decisionalità del collettivo, lo stretto rapporto insegnanti-studenti per la programmazione didattica e la gestione dei corsi. Docenti, esperti e sindacalisti collaboravano alla costruzione e conduzione delle lezioni e i contenuti forniti dagli studenti passavano attraverso un processo di discussione collettiva che li coinvolgeva in prima persona, evitando l'accumulo di nozioni e fornendo la percezione di una cultura utile alla comprensione della realtà. L'utilizzo di metodi democratici nell'ideazione come nella gestione dei corsi traeva spunto dall'esperienza dei collettivi studenteschi e dei consigli all'interno delle aziende e il lavoro di gruppo assumeva un ruolo caratterizzante. Il tentativo era quello di introdurre modalità di apprendimento alternative, senza tramutare le 150 ore in un'esperienza "speciale" avulsa del tutto dal contesto scolastico e ridotte ad una sorta di ghetto, seppur progressista ed avanzato. Un'attenzione particolare fu attribuita soprattutto alla ricerca. "Non abbiamo fatto una scuola tradizionale, ma una ricerca" oppure "la scuola ce la siamo fatta da noi da soli perché abbiamo fatto una ricerca e non studiato sui libri", sono interventi comuni di lavoratori corsisti nei quali di solito prevaleva la valutazione positiva di quell'esperienza nuova che passò con l'etichetta di "ricerca". Scorrendo l'archivio Cisl sulle 150 ore $^{5}$ è possibile imbattersi in una moltitudine di materiali: analisi sull'emigrazione in Italia (b. 13862 fasc. 6), sullo sviluppo economico ed industriale (b. 13890 fasc. 3.2), sulla storia dei partiti politici (b.13876 fasc. 3) e il movimento sindacale (b. 13879 fasc.14, b.13980 fasc.3.1), su fascismo e Resistenza (13879/7), numerose inchiesta sui problemi delle operaie turniste (es: "Ricerca sulla Fabbrica" delle operaie del Tubettificio Ligure di Lecco (b.13846 fasc.1). Una grande mole di materiale è dedicata all'analisi della salute e sicurezza nei luoghi di lavoro. A titolo di esempio il fascicolo 1341/1 contiene dispense su "Ambiente di lavoro e salute" prodotte da gruppi di lavoratori di diverse aziende di Genova (Italsider, Ansaldo, Nuova San Giorgio) con la collaborazione di studenti di Medicina: emblematico esempio del nuovo atteggiamento sindacale nei confronti della professionalità che non

\footnotetext{
${ }^{5}$ Il Fondo 150 ore della Biblioteca Centrale Cisl rappresenta quanto di
} cartaceo la Flm conservava sullesperienza delle 150 ore. 
poteva essere valorizzata se non miglioravano le condizioni in cui esso veniva esercitato (Causarano 2015).

Tematiche nuove affrontate con strumenti didattici alternativi al libro di testo: il lavoro di gruppo, l'inchiesta, l'uso della statistica e del quotidiano ma anche il teatro come testimonia un articolo sugli operai metalmeccanici di Pontedera (Cervi 1978) e restituito in tutta la sua evidenza dall'esperienza del teatro operaio a Terni.

\section{"LA SCENA INCOMINCIÒ AD AVERE L'EFFICACIA DI UN INSEGNAMENTO»: GLI OPERAI DI TERNI E IL TEATRO DI BRECHT}

Terni 25 gennaio 1975.

Sta per concludersi a Terni il seminario teatrale incentrato su "L' eccezione e la regola" di Bertolt Brecht che ha visto al lavoro per circa due settimane, settantacinque operai delle Acciaierie di Terni, oltre ad una ventina di studenti e attivisti dei gruppi teatrali di base che operano in Umbria. L'iniziativa, voluta dagli operai della Terni che hanno inteso utilizzare in questo modo le 150 ore ottenute con il contratto dei metalmeccanici (le ore che la società paga e che i dipendenti spendono per istruirsi), è stata organizzata dagli enti locali, primo tra tutti la regione Umbria, dalle organizzazioni del tempo libero, e dal Consiglio di Fabbrica della Terni. Ma è chiaro che i veri protagonisti di questo seminario, unico per il tipo di partecipanti, sono gli operai della Terni che hanno avuto come animatore un regista d'eccezione: Benno Besson, sovrintendente della Volksbuhne di Berlino democratica.

(Acconciamessa 1975)

Nel 1975 i lavoratori delle acciaierie di Terni decisero di utilizzare le 150 ore per capire e fare teatro. Lo fecero discutendo con Benno Besson, direttore del Teatro del Popolo di Berlino, un'opera didascalica di Bertolt Brecht, "L'eccezione e la regola", di cui stesero le note di regia e che misero in scena in una rappresentazione di fabbrica.

Si trattava di un utilizzo assolutamente originale del nuovo istituto contrattuale che acquisiva un significato ancora più profondo alla luce delle finalità del dramma didattico di Brecht: attivare la partecipazione degli operai attraverso il teatro. Tra il 1928 e il 1934 Brecht si dedicò alla stesura dei cosiddetti "drammi didattici", testi in contrapposizione a tutte quelle forme di manifestazione sceniche finalizzate ad intrattenere ma non a far pensare lo spettatore. Brecht riteneva infatti che il teatro dovesse avere una funzione pedagogica, passando così da una funzione di puro divertimento, ad una di insegnamento. "I filosofi borghesi fanno una grossa differenza tra chi agisce e chi osserva. L'uomo che pensa non fa tale differenza [...] bisogna educare i giovani per mezzo della recitazi- one teatrale, vale a dire, facendone persone che agiscono e osservano al tempo stesso» (Brecht 1975, 73). Ispirato dal marxismo, Brecht elaborò una sua ben precisa teoria drammaturgica considerando come primo "destinatario" del suo teatro il proletariato, che doveva, attraverso la riflessione sulle vicende rappresentate, educarsi alla critica storico-politica. Una volta stabilito questo "scopo" il drammaturgo tedesco giunse alla concezione di un teatro nuovo che definì "teatro epico" fatto con persone comuni prese dalle fabbriche, dalle scuole, senza alcuna impostazione accademica alle spalle e realizzato nei luoghi della quotidianità ordinaria: manifatture ed aziende, scuole, strade e negozi (Lazzari 1977, Ronchi 2013).

"Leccezione e la regola" nacque con questi medesimi scopi. Scritto nel 1930 narra la storia di un mercante che, durante una spedizione in compagnia di un facchino di cui diffida, scambia la borraccia che questi gli offre per una minaccia, e ritenendolo malintenzionato, lo uccide. Portato a processo viene assolto: il giudice non lo condanna perché, essendo ricco e potente, aveva ogni diritto di temere persone di una classe sociale inferiore e di conseguenza l'omicidio era giustificato, indipendentemente dalla reali intenzioni del servitore. Il dramma termina con un'esortazione: "Avete ascoltato e avete veduto/ ciò che è abituale, ciò che succede ogni giorno/ Ma noi vi preghiamo: anche quello che ormai è consueto, trovatelo strano!». Un invito dell'autore a mettere in discussione quello che sembra eterno ed immutabile e a denunciare con coraggio le ingiustizie e i soprusi per uscire dal disinteresse e dal qualunquismo ${ }^{6}$. Purtroppo a causa dell'avvento del nazionalsocialismo Brecht non riuscì a portare a termine questo progetto e "L'eccezione e la regola" non fu mai rappresentato così come il drammaturgo avrebbe voluto:

dopo l'avvento del Nazismo a partire dal '33 i drammi didattici di Brecht non sono più stati utilizzati per lo scopo per cui erano stati scritti dall'autore. Essi sono stati rappresentati come le altre opere e se sono serviti per sperimentazioni, queste sono state fatte esclusivamente da attori. Ecco perché quello che gli operai di Terni hanno fatto in questi giorni di seminario è unico e prezioso e non va assolutamente perduto (Acconciamessa 1975).

Negli anni Settanta la Società Terni aveva definitivamente cessato di essere polifunzionale ${ }^{7}$ orientando

\footnotetext{
${ }^{6}$ La dimensione pedagogica del teatro brechtiano agisce oltre che sul piano dei contenuti su quello delle modalità di rappresentazione e sui meccanismi di comunicazione teatrale. Dalle tecniche di "straniamento" all'utilizzo di procedimenti letterari propri della satira (parodia, rovesciamento ecc.), dalle tecniche di composizione del testo ad un complesso di soluzioni scenografiche, cartelli, ecc. (Giosi 2011).

${ }^{7}$ A seguito della nazionalizzazione dell'industria elettrica e l'istituzione dell'Ente nazionale per l'energia elettrica (1962) gli impianti elettrici del-
} 
la produzione esclusivamente nelle lavorazioni siderurgiche e meccaniche. Continuava a rappresentare per la città il nucleo centrale intorno al quale ruotavano i mestieri e le competenze professionali, la massiccia scolarizzazione verso gli indirizzi tecnico-industriali, lo sviluppo urbanistico e le trasformazioni ambientali (Covino 1998). All'interno della fabbrica tra il 1969 e il 1976 dominarono i grandi temi della professionalità e della ricerca di nuove forme di organizzazione del lavoro arrivando alla stipula di una nuova struttura del salario e dei livelli retributivi del personale operaio ${ }^{8}$ propedeutici all'applicazione dell'Inquadramento Unico del 1972. Attraverso quest'ultimo tutte le mansioni operaie ed impiegatizie venivano raccolte nell'ambito di otto livelli retributivi (fino a cinque per gli operai) a cui corrispondono precisi attributi professionali. Un rapporto nuovo tra operai ed impiegati che implicava necessariamente un investimento nella struttura della scuola per modificarne i meccanismi di selezione e discriminazione e realizzare le condizioni per l'effettiva mobilità della forza lavoro all'interno della scala delle qualifiche. Impegno accolto dal nuovo istituto contrattuale delle 150 ore (Causarano 2015).

$\grave{E}$ in questo contesto che si realizzò il seminario di cultura teatrale "Rinnovamento culturale e classe operaia" promosso dal Consiglio di fabbrica della Terni, insieme alla Regione Umbria, alla provincia, al Comune e ad associazioni legate al tempo libero come Endas, Arci ed Enars con lo scopo di: "contribuire alla costruzione di quella nuova cultura di cui le masse lavoratrici del nostro paese, sentono sempre di più l'inderogabile bisogno per il superamento di un modello di sviluppo culturale (e di conseguenza politico ed economico) che oggi fa ancora perno sulle barriere storiche tra fabbrica e scuola, tra fabbrica e società)» ${ }^{9}$. L' iniziativa vide la partecipazione di ottanta lavoratori ed iniziò ufficialmente il 16 gennaio presso la sala "Farini" concessa dal Comune in quanto l'azienda aveva rifiutato di svolgere gli incontri all'interno della fabbrica perché riteneva il teatro poco aderente ai reali bisogni dei lavoratori:

L'atteggiamento dell'azienda fu dapprima ambiguo, la direzione temeva che l'iniziativa fosse troppo rivoluzionaria,

\footnotetext{
la Società Terni vennero espropriati decretando la fine della polisettorialità e la nascita della nuova Terni siderurgica appartenente al gruppo Iri-Finsider.

${ }^{8}$ Accordo sindacale del 9 luglio 1969 con il quale viene sanata anche la distinzione retributiva tra operaio siderurgici e meccanici (i primi godevano di un trattamento più elevato). Per approfondimenti: Fogliano 1984

${ }^{9}$ Relazione di Gian Filippo Della Croce a nome del Consiglio di Fabbrica della Terni in apertura del seminario, Terni, 15 gennaio 1975 (Moscati 1977, 46)
}

troppo legata alla sinistra, ma la vicinanza al PSI dell'amministratore delegato e del capo del personale facilitarono alla fine il consenso della direzione che comunque lo aveva legato al consenso del consiglio di fabbrica. Lo stesso consiglio era percorso dall'entusiasmo e dal dubbio. La CISL FIM temeva che il seminario potesse diventare un'iniziativa politica in mano al PCI e alla CGIL e dapprima le sue obiezioni causarono una serie di sedute del consiglio dedicate all'argomento, dove alla fine dimostrammo che l'iniziativa doveva essere sotto l'egida del consiglio e tutte le componenti sindacali ne avrebbero fatto parte. A quel punto il consiglio all'unanimità votò per l'attuazione del seminario (intervista a Gian Filippo Della Croce).

La gestione e l'organizzazione pratica fu assunta interamente dal Consiglio di fabbrica, più precisamente dalla commissione scuola, che aveva proceduto alla raccolta delle iscrizioni e alla preparazione delle conferenze di dibattito su temi politici e culturali a corollario dell'iniziativa. Il primo dei dodici incontri fu dedicato alla lettura dell'intera opera accompagnata da momenti di discussione sui personaggi e la loro trasposizione nella realtà di fabbrica e nella società. Un'esperienza aperta anche ai cittadini e a tutti coloro che volessero portare il loro contributo in linea con il dépliant illustrativo del seminario: "Con il teatro, dalla fabbrica alla società" che rappresentava realmente le caratteristiche di impegno proposte dell'iniziativa. Con il trascorrere dei giorni e lo studio del copione il mercante, il portatore, la guida e il giudice assunsero in breve tempo «decine di fisionomie e di intonazioni vocali» (Moscati 1977, 85) riuscendo ad avere un volto e un nome ben definiti grazie ad operai dei reparti profilati, imbracatori ma anche impiegati di reparti lavorazione speciali o dell'ufficio organizzazione del lavoro:

C'era un $30 \%$ che non aveva conseguito la terza media, un $50 \%$ con diploma di terza media e un $20 \%$ erano diplomati di scuole professionali e istituti tecnici. Le mansioni che svolgevano erano diverse: operatori macchine utensili, addetti ai laminatoi, carropontisti, manutentori, provenivano principalmente dall'area delle lavorazioni a caldo che in quel momento avevano poco lavoro e così la direzione ci chiese di privilegiare la partecipazione di quegli operai. Non ci furono critiche da parte dei lavoratori sull'uso delle 150 ore, la formula dei "seminari tematici" che era prevista nell'uso di quello strumento ebbe a Terni la dimostrazione che era una scelta giusta. Nel sindacato ci fu qualche mugugno di tipo ideologico (intervista a Gian Filippo Della Croce).

Pur di fronte a gravi problemi occupazionali e di ristrutturazione ${ }^{10}$ e anche se alcuni membri del Con-

\footnotetext{
${ }^{10}$ Tra il '74 e il '75 l'azienda attraversò una crisi severa dovuta alla drastica riduzione degli sbocchi di mercato (determinati dalla crisi petroli-
} 
siglio di fabbrica sostenevano che era più opportuno partire con i corsi della scuola dell'obbligo perché «non si poteva perdere tempo e ore per un'iniziativa come quella del seminario su Brecht ${ }^{11}$, quest'ultimo ottenne una massiccia pioggia di adesioni da parte dei dipendenti. L'iniziativa sul teatro rappresentò infatti una risposta concreta ai tentativi da parte padronale di restringere l'uso delle 150 ore e di svuotarne il significato, o di utilizzarle in termini di ricomposizione o arricchimento delle mansioni, nella ricerca di una nuova forma di elasticità della forza lavoro, spingendo i lavoratori ad allargare la capacità professionali in funzione delle nuove esigenze produttivistiche. Come scrisse all'epoca Gian Filippo Della Croce:

I lavoratori della nostra fabbrica che si sono iscritti per frequentare il seminario sono la più valida di tutte le risposte a questi tentativi riduttivi, e dimostrano ancora una volta, se ce ne fosse bisogno, l'uso che intendono farne per un fine collettivo e unificante. Il seminario si inscrive infatti nelle linee fondamentali che la Flm ha indicato per le 150 ore e che prevedono oltre ai corsi per il recupero della scuola dell'obbligo, appunto seminari e corsi monografici di più ampio respiro, che si ricollegano alla linea sindacale di conoscenza, controllo e modifica dell'organizzazione del lavoro in fabbrica e di intervento fuori della fabbrica, sviluppando tutta la tematica che sta di fronte al movimento. Un uso politico di questa conquista, dunque che supera vecchi metodi di insegnamento elaborati dalla classe dominante, parte dell'esperienza reale della classe lavoratrice, dai suoi momenti di analisi, dai suoi obiettivi e dal patrimonio delle sue lotte (Moscati 1977, 46).

Il seminario ebbe il suo punto di forza nel dibattito tra regista e gli operai. Un lavoro con il teatro in cui Besson non era chiamato in veste di professore ma per partecipare insieme con i lavoratori ad una sorta di animazione politica e culturale, portando il suo contributo di studioso. Di seguito alcuni stralci della discussione che animò il laboratorio tratti da "GruTeaTerTeatro Operaio" a cura dello sceneggiatore Italo Moscati:

\section{Besson}

Tanto per cominciare, in onore di Brecht potremmo leggere il prologo del testo $[\ldots]^{12}$. E' molto significativo, per-

\footnotetext{
fera e dal restringimento del mercato siderurgico) e un altissimo livello di indebitamento legato al massiccio rinnovo impiantistico degli anni immediatamente successivi alla nazionalizzazione del settore elettrico (Butera 1979)

${ }^{11}$ Note in margine alla conclusione del seminario "Rinnovamento, culturale e classe operaia", Terni, febbraio 1975 (Moscati 1977, 79)

${ }^{12}$ Vogliamo riferirvi la storia/ Di un viaggio compiuto/ Da uno sfruttatore e da due sfruttati. / Osservatene bene il contegno.

Trovatelo strano, anche se consueto, /inspiegabile, pur se quotidiano, /
}

ciò bisognerebbe insistere perché ognuno lo ascolti bene e lo comprenda. Non è troppo facile bisogna ascoltarlo bene. Non si tratta di declamare una poesia ma di dire: qual è la vostra opinione sul prologo?

Un operaio: Brecht ci invita a guardare sotto la storia semplice di un viaggio. Ciò significa che i fatti esposti riguardano anche la nostra realtà quotidiana, e che noi non possiamo rimanere inermi.

Un operaio: Credo che Brecht con questo dramma volesse dire che nel viaggio si verificano fatti apparentemente inspiegabili. E rende possibile con questo stimolo un raffronto con la vita che stiamo attualmente vivendo.

Un operaio: Praticamente molto spesso ognuno di noi accetta come naturali delle cose che vengono fatte normalmente, e sono mostruose. Però l'importante è non accettarle passivamente ma chiedersi perché queste cose avvengono giornalmente. Sono fatti comuni, fatti semplicissimi: infatti la storia è semplice, è una storia molto semplice, ma la si può trasferire su altri piani, la si può trasferire su altri fatti. E capire.

Un operaio: C'è una cosa importante che tutti dobbiamo fare: non vedere i personaggi come attori ma pensare, per esempio, che il giudice è una figura concreta, come altre che operano nella società borghese mentre noi siamo qui a studiare Brecht. Un giudice in questo momento probabilmente sta trattando il caso di un qualsiasi proletario in un tribunale d'Italia. E contemporaneamente circolano libere figure altrettanto concrete come quelle del mercante $[\ldots]$.

Un operaio: Anche a me ha colpito la figura del mercante [...] ciò che è importante per me è vedere contrapposta alla sua regola, l'eccezione.

Besson: A mio avviso avete colto un aspetto molto importante del testo di Brecht. L'eccezione io credo è proprio quel comportamento umano che stabilisce rapporti nuovi e consente la creazione di una solidarietà tra persone che si oppongono ad una realtà sentita come condizionante. Un' eccezione, è quanto stiamo sviluppando qui tra noi [...].

(Moscati 1977, 46).

L'opera fu messa in scena appena terminato il ciclo di incontri dando vita alla spedizione del mercante Karl Langmann che attraverso il deserto cerca di arrivare alla città di Urga prima dei concorrenti per ottenere una concessione petrolifera. Gli operai non si cimentarono solo nel ruolo di interpreti ma anche di suggeri-

indecifrabile, pure se è regola. /Anche il minimo atto, / in apparenza semplice, / osservatelo con diffidenza! Investigate se/ specialmente l'usuale sia necessario. / E - vi preghiamo- quello che succede ogni giorno/ Non trovatelo naturale/Di nulla sia detto è naturale. 
tori, direttori dei cori, costumisti e musicisti. Come si legge sulle pagine de l'Unità si trattò di un lungo e faticoso impadronirsi di uno dei tanti strumenti culturali ancora estranei alle grandi masse popolari: "Anche il teatro nelle 150 ore allo stesso modo dello studio della matematica o della storia, per avere un recupero di quanto la scuola interrotta per forza non è riuscita a dare» (Melograni 1975). Il valore della vicenda e le sue ricadute in termini pedagogici sono messe ben in luce anche dalle parole di Gian Filippo Della Croce che nell'intervista ricorda come gli operai:

prima di tutto appresero il significato delle 150 ore, poi ebbero la possibilità, come mai era stato possibile prima, di potersi confrontare tra loro apertamente senza ostacoli ideologici, su argomenti che interessavano tutti. Appresero l'uso del teatro come mezzo di comunicazione e di crescita sociale e culturale, come mezzo di battaglia politica e per loro fu una grande scoperta. Appresero come poteva essere utilizzato il teatro e quello di Brecht in particolare per parlare e per riflettere sulla loro condizione e trovare punti di vista comuni. Appresero anche che potevano usare direttamente quella forma teatrale e che ne avevano le possibilità. La struttura del testo de "L'eccezione e la regola" consentiva di acquisire la capacità di comprendere fenomeni e vicende che vivevano tutti i giorni, di questo i partecipanti parlarono ampiamente nelle fasi conclusive del seminario. Un commento per tutti: "prima del seminario, non sapevo parlare".

Lo stesso Benno Besson invitò tutti i partecipanti a non disperdere quanto appreso e a portare avanti l'esperienza: «dovete dimostrare di essere diventati dei committenti culturali, far proseguire l'attività che abbiamo iniziato qui, farla rivivere nei quartieri e riprendere il discorso momentaneamente interrotto con la popolazione, con gli altri lavoratori, con tutti i cittadini» ${ }^{13}$. L'eredità del seminario non andò dispersa ed un gruppo di operai riuscì ad organizzarsi e a portare la rappresentazione dell'opera in molte altre città italiane, in Germania $^{14}$, Francia, Svizzera, Belgio e Lussemburgo. Sempre

\footnotetext{
${ }^{13}$ Benno Besson, conferenza stampa 24 gennaio 1975. In "Note in margine alla conclusione del seminario Rinnovamento, culturale e classe operaia", Terni, febbraio 1975 (Moscati 1977, 89)

14Da l'Unità del 24 febbraio 1976: «Il seminario fu replicato a Berlino nel 1976. Parteciparono un gruppo di una ventina di lavoratori delle Acciaierie Terni, operai, tecnici e impiegati tedeschi della Secura, impresa produttrice di macchine calcolatrici e della Narva, un'azienda per la fabbricazione di lampadine. Emblematiche le parole di un responsabile sindacale: da un punto di vista economico, il seminario è costato la mancata produzione di settantamila lampadine. Ma ne valeva la pena. Abbiamo imparato a conoscere meglio noi stessi e gli altri, abbiamo scoperto nuove possibilità per realizzarci in modo più compiuto come uomini. E questo fa parte di quello sviluppo completo della personalità socialista che è uno degli obiettivi di fondo del prossimo congresso del nostro Partito» (Barioli 1976)
}

facendo tesoro di quanto appreso con Besson operai, impiegati, studenti riunitisi all'interno del GruTeaTer (Gruppo Teatro Terni) condussero una ricerca che diede vita allo spettacolo "Giorni del movimento operaio" dedicato alla storia della Terni dalla fondazione avvenuta nel 1884 fino ai licenziamenti del 1953 (Acconciamessa 1976). Lo studio fu condotto sul campo intervistando operai ancora attivi o in pensione e ricostruendo pazientemente, tassello su tassello, anni e anni di battaglie, di sconfitte, di vittorie, incluso il drammatico episodio dell'operaio Trastulli, ucciso dalla polizia nel marzo 1949 durante una manifestazione contro il Patto Atlantico (Portelli 2007). Una ricostruzione storica proposta attraverso lo strumento teatrale volta a coinvolgere il pubblico invitato a dare il suo apporto:

Il nostro lavoro non vuole essere una celebrazione o un revival ma un contributo alla ricerca (che da parte nostra non si fermerà qui) sul movimento lavoratore ternano attraverso una proposta di lavoro teatrale globale. Proposta che sfruttando in modo nuovo la possibilità del mezzo, lo utilizza in un'operazione di informazione di massa su vicende ancora vive nella memoria di migliaia di lavoratori e cittadini di Terni, e di recupero del vasto patrimonio culturale operaio. Difatti siamo convinti che la drammatizzazione di parte della nostra ricerca non interrompe la ricerca anzi l'arricchisce di uno strumento in più. Si può ben dire che in "Giorni del movimento lavoratore ternano", non si recita, si ricerca collettivamente (Moscati 1977, 125).

\section{CONCLUSIONI}

L'articolo ha cercato, seppure brevemente, di ripercorrere il contributo e gli ideali di questa straordinaria esperienza che ha inciso sulla vita di numerosi operai e sindacalisti delle acciaierie Terni. Si trattò di un importante momento di sperimentazione e proposta culturale gestito interamente dal sindacato nello spirito collettivo delle 150 ore, «una verifica a livello nazionale della possibilità da parte della classe lavoratrice e del movimento sindacale, di una gestione collettiva dei mezzi di produzione culturale» (Moscati 1977, 21). Se lo scopo immediato dei lavori era verificare la proponibilità, in un contesto tanto mutato, dei drammi didattici di Brecht, il significato innegabile dell'iniziativa «fu anche nel tentativo di definire compiutamente che cosa dovevano essere le 150 ore, interpretate, nell'appuntamento ternano, come occasione di crescita culturale collettiva, senza lo sbocco obbligato della formazione di tipo strettamente scolastico e professionale» (Romagnolo 2000, 139). Secondo la testimonianza offerta a suo tempo da Besson sulle pagine de l'Unità il seminario acquisì fin da subito un carattere fortemente legato alla quotidianità dei lavoratori: 
Ciò che mi ha impressionato in quella esperienza era il concetto di cultura, così come lo intendevano i lavoratori. L'arte per loro non poteva essere considerata staccata dalle condizioni della vita. Era niente fuori di questo rapporto. Al contrario di quanto afferma la tradizione borghese. Lavoravamo intensamente sette o otto ore al giorno, tutti seguivano con attenzione e responsabilità. Hanno utilizzato dal loro punto di vista l'opera di Brecht come non mi è mai accaduto di vedere. C'è stato un lavoro migliore perfino sul piano scientifico di quanto, per esempio, ho verificato a Roma nel corso di un'altra esperienza di questo genere, condotta però con gli studenti dell'università. Qui ci fu una comprensione astratta degli strumenti elaborati da Brecht e gli stessi materiali del seminario non sono stati utilizzati come è avvenuto per Terni (Leiss 1978)

L'attualità de "L'eccezione e la regola" di Brecht emergeva dal confronto con i concreti problemi del lavoro in fabbrica, con le condizioni di vita, con la pratica effettiva della giustizia e le varie situazioni familiari, che erano parte integrante della discussione sul testo. Un laboratorio realizzato in un clima di forte partecipazione, aggregazione ed impegno civile e un nuovo modo di intendere il momento educativo, allargandolo e socializzandolo, dando cioè all'educazione una dimensione comunitaria. Una vicenda che dimostra come «l'ambiente tecnologico non sia estraneo ad aspetti umanistici» (Della Croce) indissolubilmente legati all'individuo e che consente di riflettere su dinamiche e sfide ancora assolutamente attuali per l'educazione degli adulti: la capacità della formazione di essere percepita in chiave trasformativa ma soprattutto l'accrescimento della qualità educativa nei luoghi di lavoro.

\section{RINGRAZIAMENTI}

Gian Filippo Della Croce, Sindacato FIOM CGIL, coordinatore seminario 150 ore alle acciaierie di Terni

Carla Pagani, Redazione LiberEtà

Aldo Gara, Redazione LiberEtà

\section{BIBLIOGRAFIA}

Acconciamessa, Mirella. 1976. "Come i lavoratori di Terni recuperano la loro storia”. l'Unità, 13 giugno.

Acconciamessa, Mirella. 1975. "Brecht didattico vive tra gli operai di Terni”. l'Unità, 26 gennaio.

Marzio, Barbagli, e Marcello Dei. 1969. Le vestali della classe media. Ricerca sociologica sugli insegnanti. Bologna: Il Mulino.

Barioli, Arturo. 1976. "Operai di Terni e Berlino lavorano due settimane su Brecht didattico". l'Unità, 24 febbraio.
Bernardi, Claudio. 2014. Il teatro sociale. L’arte tra disagio e cura. Roma: Carocci.

Brecht, Bertolt. 1977.Leccezione e la regola. Torino: Einaudi.

Brecht, Bertolt. 1975. Scritti teatrali I. Torino: Einaudi.

Brecht, Bertolt. 1975. La teoria del dramma didattico, in Id., Scritti teatrali III. Note ai drammi e alle regie. Torino: Einaudi.

Bini, Giorgio. 1975. Didattica delle 150 ore. Roma: Editori riuniti.

Butera, Federico. 1979. Lavoro umano e produzione. Una ricerca sulle acciaierie Terni. Einaudi: Torino.

Cavazzoni, Ermanno. 1976. Guida alla lettura del quotidiano. Lo studio dellitaliano in un corso 150 ore. Firenze-Rimini: Guaraldi editore.

Causarano, Pietro, cur. 2016. Lavoro, formazione e educazione in prospettiva storica: sollecitazioni e ipotesi per nuovi campi di ricerca. Pisa: ETS.

Causarano, Pietro. 2015. "Unire la classe, valorizzare la persona. L'inquadramento unico operai-impiegati e le 150 ore per il diritto allo studio". Italia contemporanea 278: 224-46.

Causarano, Pietro, e Zazzara, Gilda. 2015. "Le 150 ore del Veneto" in Venetica. La scuola delle 150 ore in Veneto $31 / 2015$ a. XVIII: 7-30.

Cervi, Ciro. 1978. "Con le 150 ore studiano teatro". Paese Sera, 13 febbraio.

Cornacchia, Matteo. 2018. Le humanities in azienda. Per una via umanistica alla formazione. Milano: Franco Angeli.

Covino, Renato. 1998. Le acciaierie di Terni. Firenze: Electa.

Cuppone, Roberto. 2018. Stultifera navis: teatro e follia in Italia negli ultimi quarantanni. Torino: Accademia University Press.

Demetrio Duccio, cur. 1977. 150 ore e diritto d'alfabeto. Alfabetizzazione degli adulti e realtà operaia. RiminiFirenze: Guaraldi.

De Sanctis, Filippo Maria. 1978. L'educazione degli adulti in Italia, Roma: Ed. Riuniti.

Foa, Vittorio. 1976. La struttura del Salario. Firenze: Alfani.

Fogliano, Franco. 1984. Potere, sindacato, società a Terni. 1969-1983. Terni: Edizioni Thyrus

Giachetti, Diego. 2008. Un sessantotto e tre conflitti. Generazione, genere, conflitto. Pisa: BFS edizioni.

Gigli, Alessandra, Tolomelli, Alessandro e Zanchettin, Alessandro. 2008. Il teatro dell'oppresso in educazione. Roma: Carocci.

Giosi, Marco. 2011. Come in uno specchio: teatro e formazione dell'io: figure e percorsi del Novecento. Roma: Anicia. 
Lazzari, Arturo. 1977. Letà di Brecht. Milano: Rizzoli

Gurrieri, Giovanna. 1975. Tornare a scuola da protagonisti. L’esperienza delle 150 ore. Rimini Firenze: Guaraldi Editore.

Leiss, Alberto. 1978. "Brecht un classico? Ma se è da scoprire! Benno Besson, uno dei protagonisti della storia del teatro dal dopoguerra ad oggi, racconta le sue esperienze al fianco del grande drammaturgo". l'Unità, 5 settembre.

Lettieri, Antonio. 1970. "Note su qualifiche, scuola e orari di lavoro", in Problemi del socialismo, n.49 anno XII, novembre dicembre, 809.

Levi Arian Giorgina, Alasia Giovanni, Chiesa Adalberto, Bergoglio Pietro, e Benigni Letizia. Cur.1969. Con introduzione di Foa, Vittorio. I Lavoratori Studenti. Torino: Einaudi.

Marinelli, Valerio. 2019. Il Sessantotto studentesco di Perugia e il Sessantotto operaio di Terni tra storia e memoria. Italia contemporanea 290: 68-93.

Melograni, Luisa. 1975. "Le 150 ore di teatro tra finzione e realtà", l'Unità, 5 settembre.

Moscati Italo, cur. 1977. GruTeaTer, Teatro operaio. Roma: Coines Edizioni

Pagnoncelli, Lucio. 1977. Le 150 ore, Firenze: La Nuova Italia.

Portelli, Alessandro. 2007. Storie Orali. Racconto, immaginazione, dialogo. Roma: Donzelli.

Raspadori, Paolo. 2006. "Un'eroica sconfitta: I licenziamenti alle acciaierie di Terni nei primi anni Cinquanta". Studi Storici. Anno 47, 1 (Gen - Mar): 247-81.

Raspadori, Paolo. 2017. "Nascita, successi e declino del welfare aziendale a Terni (1886-1975)". Ricerche Storiche XLVII. 1: 111-138.

Raspadori, Paolo. 2020. Nella periferia industriale. Conflitto di fabbrica in Umbria prima e dopo l'Autunno caldo: una prospettiva quantitativa in Bartolini Stefano, Causarano Pietro, e Gallo, Stefano. Un altro 1969: i territori del conflitto in Italia. Palermo: New Digital.

Romagnolo, Raffaella. 2000. "Due convegni a Rocca Grimalda". La Ricerca Folklorica. Antropologia dellambiente, 41: 137-41.

Ronchi, Rocco. 2013. Brecht. Introduzione alla filosofia. Milano: Et al.Edizioni.

Rossetti Pepe, Gabriella. 1975. La scuola delle 150 ore. Esperienze, documenti e verifiche. Milano: Franco Angeli.

Petrini, Francesco. 2018. Lucca, Memorie Vissuti e percorsi eccentrici alla scoperta del genius loci. Lucca: Tra le Righe.

Santamaita, Saverio. 2010. Storia della scuola. Milano: Mondadori
Santomauro, Gaetano. 1975. Centri sociali di educazione permanente, Bari: La Stamperia.

Scuola di Barbiana (1971), Lettera ad una professoressa. Firenze: Libreria Editrice Fiorentina.

Targhetta, Fabio. 2015. "Istruzione popolare ed educazione degli adulti in Italia. Lineamenti storici dall'unità alle 150 ore", in Venetica, La scuola delle 150 ore in Veneto, 31/2015 a. XVIII: 31-48.

Taormina, Antonio, e Valenti, Cristina. 2013. Per una storia del teatro carcere in Italia. Reti, contesti e prospettive, Economia della Cultura. 234: 441-454.

Trentin, Bruno. 1977. Da sfruttati a produttori. Bari: De Donato.

Valentini, Valentina. 1987. Teatro in immagine. Eventi performativi e nuovi media. Vol. I. Roma: Bulzoni. 